\title{
Escobar Syndrome Associated with Spine and Orthopedic Pathologies: Case Reports and Literature Review
}

\section{Balioglu MB*}

Metin Sabanci Baltalimani Bone Disease Education and Research Hospital, Istanbul, Turkey

\begin{abstract}
Escobar syndrome (ES) is associated with a web across every flexion crease in the extremities (most notably the popliteal space) and other structural anomalies such as a vertical talus, clubfoot, thoracic kyphoscoliosis and severe restrictive lung disease. In our study, we evaluated 3 patients diagnosed with multiple pterygium syndrome (MPS) type Escobar. The purpose of this study was to assess the abnormalities of the vertebrae and concomitant orthopedic pathologies. Two male patients (17 and 20-year-old siblings) and one female patient (9 year-old) were diagnosed with ES by genetic analysis. Patients had been diagnosed with kyphosis and progressive scoliosis (except one), high-set palate, ptosis, low-set ears, arachnodactyly, craniofacial dysmorphism, mild deafness, clubfoot, hip luxation, and joint contractures. Patients received operations for dislocation of the hip, clubfoot correction (except the female patient), and contractures of the knee and ankle. Furthermore, patients also underwent surgery for ptosis and inguinal hernias (except the female patient). One male patient received posterior vertebral instrumentation and fusion for a progressive spine deformity. Spinal and orthopedic pathologies commonly occur in patients with ES and scoliosis, and kyphosis may progress considerably over time. Early surgical treatment is recommended for joint contractures, hip dislocation, clubfoot deformities, and for progressive spinal deformities.
\end{abstract}

Keywords: Escobar syndrome; Multiple pterygium syndrome; Scoliosis; Kyphosis

Abbreviations: ES: Escobar Syndrome; MPS: Multiple Pterygium Syndrome; CVT: Congenital Vertical Talus; PEV: Pes Equinovarus; AMC: Artrogripozis Multiplex Congenita; MRI: Magnetic Resonance Imaging; EOS: Early Onset Scoliosis; CEOS: Classification of Early Onset Scoliosis; USG: Ultrasonography

\section{Introduction}

Escobar syndrome (ES) is an inherited syndrome characterized by web-form skin structures and joint contracture in the extremities, particularly in all areas of flexion and folding (knee, elbow, intercrural space, and neck), most notably in the popliteal space. This syndrome may be observed together with other structural anomalies; it is frequently associated with congenital vertical talus (CVT), pes equinovarus (PEV), camptodactlia (finger contractures), cleft palate, micrognathia, ptosis, short stature, serious restrictive lung disease, and backbone deformities (thoracic kyphosis/kyphoscoliosis, lumber lordoscoliosis) [1-7]. Scoliosis is particularly common among multiple pterygium syndrome (MPS) patients. Progressive scoliosis and a high incidence of intraspinal anomaly are observed together with vertebral anomalies in MPS children [1,6]. Fusion may frequently occur in the cervical area. While its etiology is not clear, it suggests an autosomal recessive inheritance. In recent studies, mutation of acetylcholine receptors (CHRNG) in the embryonal subunit have been shown, and lethal and Escobar variants of MPS have been defined [8,9]. Early surgical treatment may be required due to serious backbone deformities, curve progression, restrictive lung disease, and the need for complex salvage procedures [3]. Our study aimed to present the vertebral anomalies and other structural orthopedic deformities as a result of genetic analyses in 3 patients diagnosed with Escobar type MPS.

\section{Case Report}

\section{Patient 1}

The first case, a 17-year-old male patient whose brother also had Escobar syndrome. His eldest sibling was diagnosed with arthrogyposis multiplex congenita (AMK). This patient's first anomaly was noticed under ultrasonography in the $3^{\text {rd }}$ month of pregnancy where the fetus was unable to flex the knees and was lying straight; genetic analysis showed autosomal recessive inherited ES in both siblings. The following symptoms were noted: progressive scoliosis, kyphosis, ptosis, mild deafness, facial cranial dismorphism, low-set ears, low-set hairline, micrognathia, joint contractures (axilla, elbow, hip, knee, ankle, neck), along with limitation of movement, hip dislocation, multiple pterygiums, vertical talus, camptodactlia, and hypertrophy of gingiva. Magnetic resonance imaging (MRI) revealed cauda equina at L3 (tethered chord). The patient went through various surgical operations, which included bilateral CVT (one of them twice, and bilateral resection of the navicular bone was performed), open reduction and pelvic osteotomy for hip dislocation, and unilateral inguinal hernia. Due to the progression of kyphoscoliosis, instrumentation and fusion were planned from the posterior. The preoperative scoliosis curve was $78^{\circ}$ (T5-L3) and thoracic kyphosis was $70^{\circ}$. As a result of posterior spinal correction and fusion between postoperative T3-L1 vertebrae, the scoliosis curve was corrected by $42.3 \%$ to a $33^{\circ}$ (T5-L3) curve in the early period. Lateral kyphosis angles were measured as $36^{\circ}$ (T1$\mathrm{T} 3)$ and $53^{\circ}(\mathrm{T} 3-\mathrm{L} 1)$ respectively. The implants were removed in the $3^{\text {rd }}$ post-operative year after fusion due to skin problems. Difficulties were particularly noted during anesthetic interferences. A follow-up period of 6 years revealed that the correction rate was maintained. At the last check-up, the patient was able to attend school and live an independent life (Figure 1).

\section{Patient 2}

A 20 years old male patient whose brother also had ES. In addition to experiencing similar findings to his brother, he also had CVT on one foot, PEV on the other, and a kyphotic deformity; however, he had

*Corresponding author: Mehmet Bulent Balioglu, Metin Sabanci Baltalimani Bone Disease Education and Research Hospital, Istanbul, Turkey, Tel: 90212323 707; E-mail: mehmetbalioglu2003@yahoo.com

Received February 28, 2015; Accepted April 01, 2015; Published April 05, 2015

Citation: Balioglu MB (2015) Escobar Syndrome Associated with Spine and Orthopedic Pathologies: Case Reports and Literature Review. Hereditary Genet 4: 145. doi:10.4172/2161-1041.1000145

Copyright: () 2015 Balioglu MB. This is an open-access article distributed under the terms of the Creative Commons Attribution License, which permits unrestricted use, distribution, and reproduction in any medium, provided the original author and source are credited. 

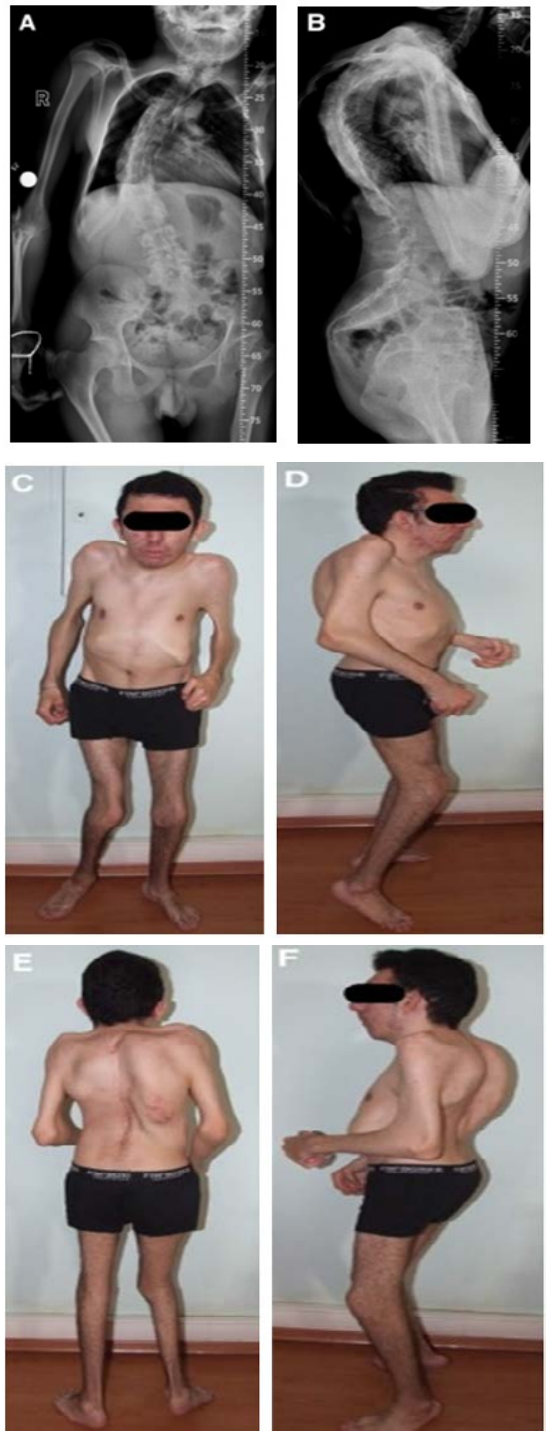

Figure 1: The first case, a 17-year-old male patient whose brother also had Escobar syndrome. Progressive scoliosis, kyphosis, ptosis, mild deafness facial cranial dismorphism, low-set ears, low-set hairline, micrognathia, join contractures (axilla, elbow, hip, knee, ankle, neck); limitation of movement, hip dislocation, multiple pterygiums, vertical talus, camptodactlia, hypertrophy of gingiva, and tethered cord were identified. The surgical treatments of the patient included bilateral CVT and hip dislocation for open reduction, and inguinal hernia. Due to the progress of the kyphoscoliosis deformity, he was treated with posterior spinal instrumentation and fusion. The instrumentation was removed due to problems caused by the implant in the third postoperative year. Radiographic image of the spinal deformity in the coronal (A) and sagittal plane (B). The appearance of the final examination, coronal and sagittal clinical pictures ( $, D, E$, and $F)$.

no scoliosis. He underwent surgery in his early childhood for inguinal hernia, CVT, PEV phythosis, and hip dislocation. The first diagnosis was set as arthrogryposis multiplex congenita. An ES diagnosis was only established as a result of a genetic inspection when his brother was born. The identified anomalies were: inability to flex knees, inability to grip objects with hands, failure to lean forward (contractures on the hips and knee joints, restriction in forward flexing movements of the backbone), low-set hairline, low-set ears, slight loss of hearing, facial cranial dismorphism, microgranthia, phythosis, high-set palate, joint contractures (axilla, knee, hip, elbow, ankle), multiple pterygiums (axilla, elbow, knee), hand anomalies (camptodactlia, arachnodactlia), vertebra anomalies (kyphosis), inguinal hernia, bilateral hip movement restriction, and foot deformity (PEV on the right foot, CVT on the left foot). The patient underwent various operations for the ES associated pathologies. Surgery for bilateral foot deformity was performed in his $6^{\text {th }}$ and $12^{\text {th }}$ month, for left inguinal hernia at 2 months and 2.5 years of age, and for left eye ptosis. The patient went through open reduction and pelvic osteotomy at 6 and 7 years of age for hip dislocation. Problems experienced by the patient after surgical operations include revision on one hip, reoperation of the inguinal hernia, and temporary paralysis on the femoral nerve after quadricepsplasty. A brace which reinforces the knee extension mechanism with lumbar support was used for rehabilitation. Detailed analysis took place on his backbone. It was observed that the onset lateral Cobb angle of $83^{\circ}$ (C7-T6) did not change throughout nearly 6 years of follow up. Due to the patient's increased thoracic kyphosis together with a reduction in the patient's backbone flexion contracture and lumber lordosis, the center of gravity in the sagittal plane (C7-Plumb Line) was $6.7 \mathrm{~cm}$ beyond the sacrum. Upon the assessment of scoliosis in the coronal plan, the Cobb angle was measured as $3^{\circ}$ between T7 and L4. In terms of coronal balance, it was observed that the vertical line from $\mathrm{C} 7$ to the floor remained $2.7 \mathrm{~cm}$ on the left of the central sacral line, and there was no evident disruption on coronal balance. A $2 \mathrm{~cm}$ pelvic tilt to the left was identified. MRI showed a $7 \mathrm{~mm}$ diameter syringomyelia on the medulla spinalis between T8-10, reduction of cord front and back diameter in the upper dorsal kyphosis area, and posterior fusion at the level of T8-9-10 vertebrae. The patient underwent observation without any intervention for backbone problems and currently lives with limited movement (Figure 2).

\section{Patient 3}

A nine-year-old girl was diagnosed with ES as a result of genetic inspection following a consanguineous marriage. The accompanying anomalies were joint contractures (axilla, elbow, hip, knee, ankle), restriction in spinal movements, low-set hairline, low-set ears, facial cranial dismorphism, micrognathia, pythosis, high-set palate, multiple pterygiums (axilla, elbow, knee), hand anomalies (campodactlia, arachnodactyly), progressive scoliosis, inguinal hernia, and foot deformity (bilateral CVT). Patient received operations for dislocation of the hip and CVT. Onset AP Cobb angle was $48^{\circ}$ (T5-T10) and it increased by $20^{\circ}$ at the 1 year follow-up to reach $67^{\circ}$. In the sagittal plan, thoracic kyphosis and lumbar lordosis remained unchanged at $8^{\circ}$ (T2-T12) and $64^{\circ}$ (L1-S1), respectively. The patient was classified as $\mathrm{S} 3(-) \mathrm{P}^{2}$ according to the newly defined classification of early-onset scoliosis (C-EOS) [10]. No evident disruption was observed in coronal and sagittal balance. Surgery was planned using a growth-friendly implantation system due to early-onset scoliosis EOS; (Figure 3).

\section{Discussion}

Although it is known that MPS generally results from autosomal recessive inheritance, its etiology has not been completely clarified. In recent studies, mutation of acetylcholine receptors (CHRNG) in the embryonal subunit have been shown, and lethal and Escobar variants of MPS have been defined $[8,9]$. Prenatal diagnosis and genetic analyses of MPS were then made and correct approaches for clinical diagnosis and treatment became possible [11]. From an orthopedic view-point, MPS is a congenital anomaly frequently observed in the upper extremities, particularly in the popliteal area, characterized by pterygia (webbing), joint contractures evident at flexion layers, and a close association with thoracic kyphoscoliosis, lordoscoliosis, and serious respiratory distress. Its other variable characteristics are intrauterine death, facial cranial dismorphism, short stature, ptosis, low-set ears, arachnodactyly, cryptorchism, KVD, cleft lip/palate, and nail anomalies. Although 

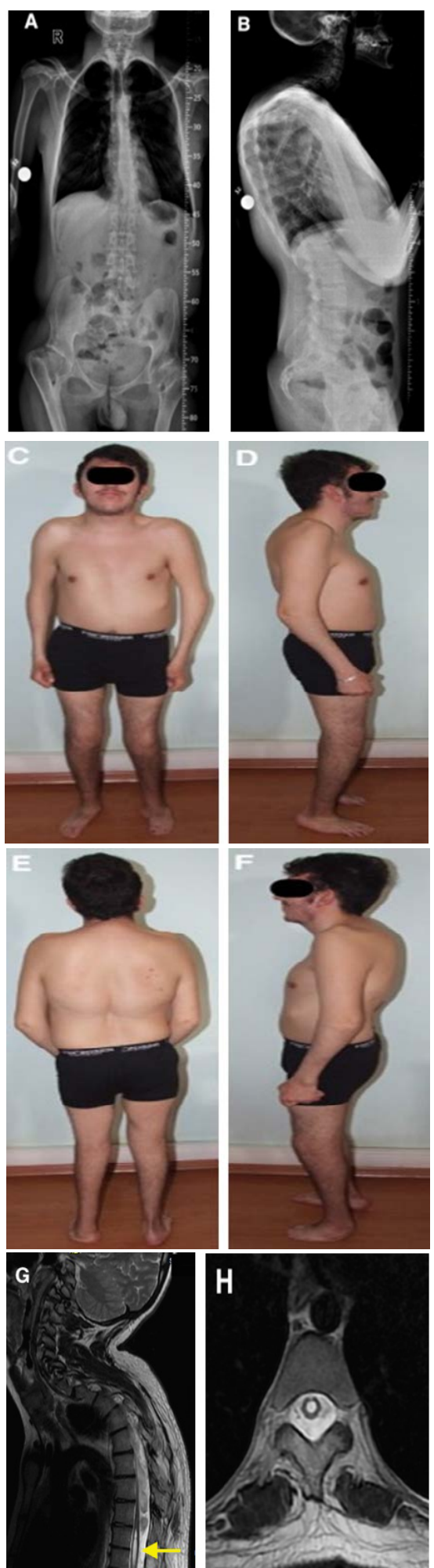

Figure 2: The second case, a 20-year-old male with accompanying kyphosis. CVT was present on one foot, PEV on the other, along with the kyphosis; however, he had no scoliosis. The patient underwent surgery in early childhood for inguinal hernia, CVT, PEV phythosis, and hip dislocation. Radiographic images of the spinal deformity in the coronal $(A)$ and sagittal plane (B). The appearance of the final examination, coronal and sagittal clinical pictures $(C, D, E$, and $F)$. Syringomyelia was observed in sagittal $(G)$ and axial sections $(\mathrm{H})$ on MRI. appearances may be normal at birth, the progress of pterygias is observed with growth.

\section{Ankle echinus contracture, PEV, and vertical talus}

Although KVT is a rarely observed foot deformity, it is commonly observed in MPS patients [1,7]. Laudrin recommended naviculectomy on children with serious middle foot deformity [7]. Angsanuntsukh found bilateral KVT in 10 out of 14 patients according to clinical data from 1969 to 2009, and emphasized that KVT (71\%) and PEV (14\%) were the most frequently noted foot deformities in MPS [1]. He detected these deformities predominantly in girls $(80 \%)$ and performed surgery as 7 of the 10 patients were ambulatory; good results were achieved with single stage surgical loosening and reduction following single stage plaster application [3]. Posterior loosening is recommended with evident contractures. Tibial shortening may be applied to prevent tensioning of neurovascular structures. Knee-ankle-foot orthesis is suggested to prevent recurrence following a period of 6-10 weeks in plaster in a knee extension. Two of our patients also had vertical talus and PEV deformities. In our first case with a more serious foot deformity, both feet were operated upon; resection of the navicular bone was performed to correct CVT deformity on one foot as other methods remained insufficient, while surgical correction and reduction were performed on two of our patients.

\section{Congenital hip dislocation}

Open reduction and pelvic osteotomies may be required to obtain a stable hip articulation. All our 3 patients underwent surgery for hip dislocation in early childhood.

\section{Upper extremities, hands}

Grasping, catching and holding functions may be reduced in these patients. Rehabilitation is initially recommended rather than surgical treatment. The brothers had limited grasping functions, however, the female patient showed better hand control.

\section{Quadriceps function, knee stability}

Inequality in leg length should be evaluated. Pterygia may be observed in the flexion region of many joints (neck 85\%, popliteal zone $60 \%$ ). Subcutenal band; a subcutaneous fibrosis band may appear extending from the ischium to the calcaneus (together with the tibial branch of the sciatic nerve). Its treatment is more difficult than other congenital knee contractures, and treatment is contraindicated if quadriceps function is lost. Treatment of equinus deformity is required to prevent recurrence. Aggressive treatments should be avoided to prevent disruption of ambulation. Conservative treatment may be applied at $30-40^{\circ}$ with serial manipulation, plaster and orthesis, and if these are insufficient, with distal femoral extension osteotomy or anterior physial tethering (steps, screws, or plate). If the flexion contracture is more than $60^{\circ}$ then complete posterior loosening, femoral shortening, and if required, anterior patellofemoral joint debridement and quadriceps or patellar tendon shortening may be performed. Surgical treatment is compulsory on popliteal pterygium patients. Opening posterior skin with Z-plasty and careful dissection of the ischiochalchaneal chord (generally wrapped with sciatic nerve branches) may be performed. The success of external fixator treatment is lower than that of other contractures. For all cases, quadricepsplasty was implemented for both lower extremities, in addition to loosening procedures for popliteal and ankle contractures.

\section{Spinal deformities}

Intraspinal anomalies and other anomalies which concern the 

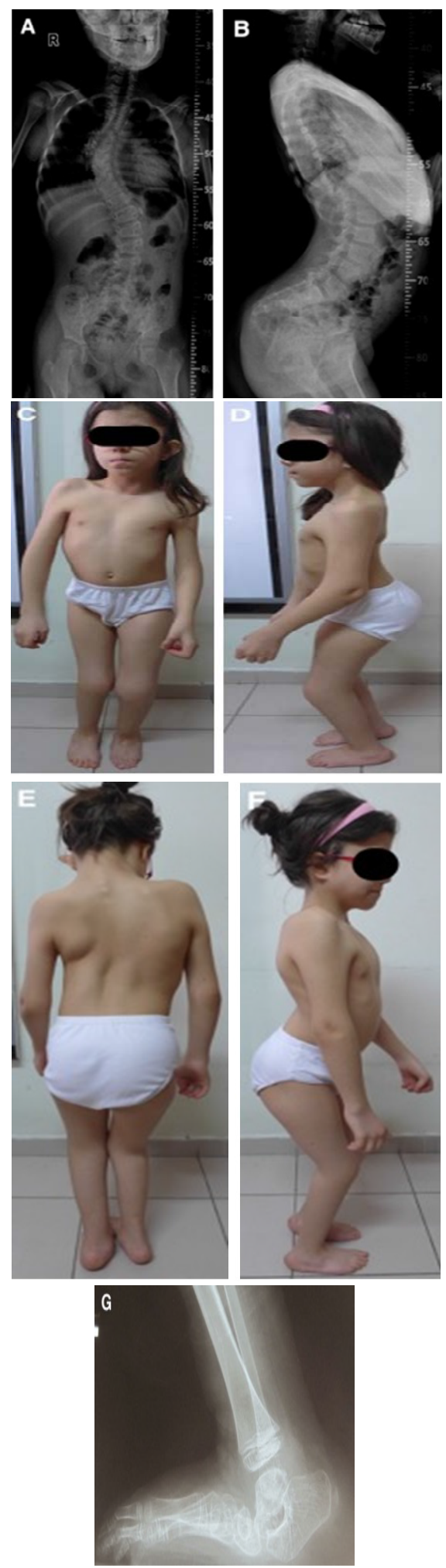

Figure 3: The third case, a 9-year-old female patient. The accompanying anomalies were joint contractures, restriction in spinal movements, low-set hairline, low-set ears, facial cranial dismorphism, micrognathia, pythosis, high-set palate, multiple pterygiums, hand anomalies, progressive scoliosis, inguinal hernia, and bilateral CVT. Radiographic image of the progressive scoliosis deformity in the coronal (A) and sagittal plane (B). The appearance of the final examination, coronal and sagittal clinical pictures (C,D,E, and $F$ ). Congenital vertical talus was observed on both feet (G) bone structure of the spine were observed together with scoliosis in MPS patients. Hall defined 11 patients in a comprehensive literature review [12]. Tsou indicated scoliosis in 29 of 48 patients (60\%) [5]. Angsanuntsukh observed scoliosis in 13 of 14 MPS patients (93\%) [1] These pathologies were observed together with intraspinal anomalies, and tethered cord was identified in 2 patients (14\%) and syringomyelia on one patient $(7 \%)$. However an exact ratio could not be provided as the MRI results of all patients were not available. In both cases, unilateral unsegmented bar was found, and during the radiological evaluation for scoliosis, the cobb angle was measured as $35.9^{\circ}(20-75)$. Joo et al., reported that the scoliosis observed in MPS children together with vertebral anomalis was progressive [6]. They noted intraspinal anomalies in 8 patients with MRI, and scoliosis in 13 of the 16 patients $(81.3 \%)$. The age at which scoliosis was identified for the first time was $3.3 \pm 2.6$ years (range, 1 month to 8.2 years). The onset cobb angles were $37.4^{\circ} \pm 18.1^{\circ}$ $\left(14^{\circ}-75^{\circ}\right)$, and they reached $43.3^{\circ} \pm 19.1^{\circ}\left(20^{\circ}-72^{\circ}\right)$. Progression was observed in the cases with scoliosis curvature. Congenital scoliosis was observed in 7 of the patients (unilateral unsegmented bar in 3 cases, fusion of cervical spines in 3 cases, and block vertebra in one case), and neuromuscular scoliosis in one case. The most commonly observed radiological finding was defined as a reduction in intervertebral disk space together with high-set vertebra in the thoracic area. Intraspinal anomalies were observed in 4 patients ( 3 tethered spinal chord, one syrinks). At the final follow-up, 5 of the 13 patients were observed as independently attending school. Scoliosis is common in MPS; it is mostly noted with cervical fusion, and intraspinal anomalies such as tethered chord and syringomyelia [6]. Fusion and unsegmentation of vertebras are indicated as the most common anomalies in MPS patients $[6,12-14]$. However the underlying pathologies could not be clearly understood. A partial explanation was attempted through a reduction of fetal movement or fetal aquinesia [8,9]. The formation of cartilage or fibrosis bar were indicated as the other reasons [15]. Contrary to the previous studies, fusion and nonsegmentation of the vertebra were revealed as common anomalies, and shrinkage in the intervertebral area, irregularity of endplates, and a reduction of vertebral height in the thoracic area were identified in the patients [6]. Similar findings were also defined by Chen; in his study on 5 MPS patients, an evident fusion on the cervical spine, narrowing on the disk site, and reduction of thoracic vertebra height were observed [16]. It was reported that the long C-shape curves, congenital curves, and the curves noted at a young age showed quick progression. We also identified fusion in the vertebra height in the thoracic area, a reduction in intervertebral disk space, and fusion in the proximal thoracic zone in the first two cases. Progressive scoliosis $\left[\mathrm{S} 3(-) \mathrm{P}^{2}\right]$ was observed in the third case, while kyphosis deformity and additional spinal anomaly were not identified, unlike in the other two cases. Particularly in MRI studies, the ratio of intraspinal anomalies are high with increased thoracic kyphosis which accompanies scoliosis $[17,18]$. The most common intraspinal anomaly observed with scoliosis is syringomyelia, either on its own or together with CM [19]. Anomalies associated with tethered cord include lipoma and diastematomyelia or fatty filum. In congenital scoliosis, intraspinal anomaly percentages increased up to $20-58 \%$ [20,21]. Joe identified intraspinal pathologies in 4 MPS patients $(30.8 \%$; 3 tethered cord, one syringomyelia) [6]. Using MRI we also imaged tethered chord (Case 1) and syringomyelia (Case 2) with ES and kyphosis deformities (Table 1).

\section{Conservative and surgical treatment of the spine:}

MPS patients receive plaster treatment until they are suitable for surgery so as not to cause any complications due to rigid spinal deformities. It has been reported that bracing and plastering cannot prevent progression. Plaster treatment for EOS has become popular in recent years. For a patient with ES and serious scoliosis deformity, 


\begin{tabular}{|c|c|c|c|c|c|c|c|}
\hline $\mathbf{N}$ & Sex & Age (y) & $\begin{array}{l}\text { Spine } \\
\text { Deformity }\end{array}$ & MRI & Orthopedic pathologies & Other pathologies & Treatment \\
\hline 1 & M & 17 & $\begin{array}{l}\text { Scoliosis and } \\
\text { Kyphosis }\end{array}$ & $\begin{array}{l}\text { Tethered cord, } \\
\text { Intervertebral fusion }\end{array}$ & $\begin{array}{l}\text { Joint contractures, hand } \\
\text { anomalies (arachnodactyly), CHD, } \\
\text { CVT, radio-ulnar synostosis }\end{array}$ & $\begin{array}{l}\text { Facial anomalies (hypertelorism, } \\
\text { cleft palate, micrognathia, low- } \\
\text { set malformed ears, pytosis), } \\
\text { slight hearing loss }\end{array}$ & $\begin{array}{l}\text { Posterior spinal fusion, open reduction of } \\
\text { hip and pelvic osteotomy, open reduction for } \\
\text { CVT (naviculectomy), surgery for pytosis and } \\
\text { hernia, releasing of stiff joint (ankle popliteal } \\
\text { and hip contractures) }\end{array}$ \\
\hline 2 & M & 21 & Kyphosis & $\begin{array}{l}\text { Syringomyelia, } \\
\text { Intervertebral fusion }\end{array}$ & $\begin{array}{l}\text { Joint contractures, multiple joint } \\
\text { pterygium, hand anomalies } \\
\text { (camptodactyly, arachnodactyly), } \\
\text { CHD, foot deformities (PEV, CVT), } \\
\text { radio-ulnar synostosis }\end{array}$ & $\begin{array}{l}\text { Facial anomalies (hypertelorism, } \\
\text { cleft palate, micrognathia, low- } \\
\text { set malformed ears, pytosis), } \\
\text { slight hearing loss, inguinal } \\
\text { hernia }\end{array}$ & $\begin{array}{l}\text { Bilateral open reduction of hip and pelvic } \\
\text { osteotomy, open reduction for CVT and PEV, } \\
\text { releasing of stiff joint (ankle popliteal and } \\
\text { hip contractures), quadricepsplasty, inguinal } \\
\text { hernia, surgery for pytosis and hernia }\end{array}$ \\
\hline 3 & $\mathrm{~F}$ & 9 & Scoliosis & No Pathologies & $\begin{array}{l}\text { Joint contractures, hand } \\
\text { anomalies, CHD, CVT, radio-ulnar } \\
\text { synostosis }\end{array}$ & $\begin{array}{l}\text { Facial anomalies (hypertelorism, } \\
\text { cleft palate, micrognathia, low- } \\
\text { set malformed ears, pytosis) }\end{array}$ & $\begin{array}{l}\text { Bilateral open reduction of hip and pelvic } \\
\text { osteotomy, releasing of stiff joint (ankle } \\
\text { popliteal and hip contractures), Bilateral open } \\
\text { reduction for CVT }\end{array}$ \\
\hline
\end{tabular}

PEV: Pes Equina Varus; CHD: Congenital Hip Dislocation; CVT: Congenital Vertical Talus; MRI: Magnetic Resonance Imaging.

Table 1: Clinical and radiological characteristics of the 3 Escobar syndrome cases.

the following treatments are necessary: a multilevel osteotomy with anterior and posterior surgery, rib resection and debridement for injury necrosis which developed postoperatively after vertebrectomy, urinary system infection treatment, and tracheostomy for persistent atelectasia. After a three-year follow-up period, the patient recovered without any neurological issues and an evident increase was obtained in the patient's respiratory functions [13]. Early surgical treatment may be required due to serious backbone deformities in ES (kyphosis/ kyphoscoliosis), curve progression, restrictive lung disease, and the need for complex salvage procedures [3]. According to a recent comprehensive study related to MPS and scoliosis, it was concluded that there were insufficient data to determine the ideal timing or type of spinal surgery [6]. Surgical timing and technical recommendations remain insufficient due to limited experience with scoliosis surgery related with MPS cases in the literature. Due to the quick progression in the scoliosis curvature we observed during our final case.

\section{Anesthesia and reanimation}

Anesthesia practices may cause serious issues for ES patients. It is advised that MPS children should be monitored closely during anesthesia due to acetyl choline receptor subunit anomalies and malign hyperthermia [6,22]. Dodson suggests evaluation by a pediatrician, pulmonologist, or otolaryngologist before surgery on these patients [13]. The most commonly observed postoperative complications on ES patients are recurrence of deformities, injury problems, epiphysial plate damage, insufficient shortening, and short segment instrumentation. Furthermore, attention should also be paid to respiratory issues, and cardiac and gastrointestinal problems $[1,3]$. The probability of malign hyperthermia during anesthesia should not be ignored [22]. Our patients faced intubation problems, particularly during anesthesia. Malign hyperthermia did not develop.

ES patients should be monitored and treated closely for orthopedic and spinal problems beginning from birth. Medulla spinalis and the entire vertebral column should be evaluated, and the surgical treatment of scoliosis and kyphosis must carefully be planned. In patients with completed spinal growth, posterior long level instrumentation and growth-friendly systems of EOS may be preferred. When needed, vertebral osteotomy and spinal instrumentation and fusion must be accompanied by neuromonitarization under intensive care conditions. Experience related to the approaches for treatment of these patients is limited as they are rarely observed.

The prevalence of scoliosis in MPS patients was found to be $81.3 \%$. The most commonly observed anomalies have been shown as narrowing down of intervertebral disk space, irregularity of endplates, and reduction in the height of thoracic vertebrae. Fusion and unsegmentation of vertebrae on MPS patients are the most common anomalies. In addition to skeletal system anomalies, ES patients with MPS including scoliosis and/or kyphosis deformities require close monitoring of neurological functions, bowel and bladder functions, and their capacities of walking and sustaining their lives independently. Bracing and plastering methods may be insufficient for preventing scoliosis curvature and early surgical treatment is recommended in such circumstances. The treatment of spinal deformities together with intraspinal anomalies should be planned very carefully and anesthetic challenges should not be forgotten. Before starting treatment, related departments should be consulted to evaluate the patient from a genetic and developmental neurology aspect.

\section{References}

1. Angsanuntsukh C, Oto M, Holmes L, Rogers KJ, King MM, et al. (2011) Congenital vertical talus in multiple pterygium syndrome. J Pediatr Orthop 31: 564-569.

2. Escobar V, Bixler D, Gleiser S, Weaver DD, Gibbs T (1978) Multiple pterygium syndrome. Am J Dis Child 132: 609-611.

3. Dodson CC, Boachie-Adjei O (2005) Escobar Syndrome (Multiple Pterygium Syndrome) Associated with Thoracic Kyphoscoliosis, Lordoscoliosis, and Severe Restrictive Lung Disease: A Case Report. HSSJ 1: 35-39.

4. Fryns JP, Volcke $P$, van den Berghe $H$ (1988) Multiple pterygium syndrome type Escobar in two brothers. Follow-up data from childhood to adulthood. Eur J Pediatr 147: 550-552.

5. Hall JG (1997) Arthrogryposis multiplex congenita: etiology, genetics, classification, diagnostic approach, and general aspects. J Pediatr Orthop B 6: 159-66.

6. Joo S, Rogers KJ, Donohoe M, King MM, Kumar SJ (2012) Prevalence and patterns of scoliosis in children with multiple pterygium syndrome. J Pediatr Orthop 32: 190-195.

7. Laudrin $P$, Wicart $P$, Seringe $R$ (2007) [Resection of navicular bone for severe midfoot deformity in children]. Rev Chir Orthop Reparatrice Appar Mot 93: 478485.

8. Hoffmann K, Muller JS, Stricker S, Megarbane A, Rajab A, et al. (2006) Escobar syndrome is a prenatal myasthenia caused by disruption of the acetylcholine receptor fetal gamma subunit. Am J Hum Genet 79: 303-312.

9. Morgan NV, Brueton LA, Cox P, Greally MT, Tolmie J, et al. (2006) Mutations in the embryonal subunit of the acetylcholine receptor (CHRNG) cause lethal and Escobar variants of multiple pterygium syndrome. Am J Hum Genet 79 : 390-395.

10. Williams BA, Matsumoto $\mathrm{H}$, McCalla DJ, Akbarnia BA, Blakemore LC, et al (2014) Development and initial validation of the Classification of Early-Onset Scoliosis (C-EOS). J Bone Joint Surg Am 96: 1359-1367. 
11. Chen CC (2012) Prenatal diagnosis and genetic analysis of fetal akinesia deformation sequence and multiple pterygium syndrome associated with neuromuscular junction disorders: A review. Taiwanese Journal of Obstetrics and Gynecology 51: 12-17.

12. Hall JG, Reed SD, Rosenbaum KN, Gershanik J, Chen H, et al. (1982) Limb pterygium syndromes: a review and report of eleven patients. Am J Med Genet 12: $377-409$

13. Ramer JC, Ladda RL, Demuth WW (1988) Multiple pterygium syndrome. An overview. Am J Dis Child 142: 794-798.

14. Thompson EM, Donnai D, Baraitser M, Hall CM, Pembrey ME, et al. (1987) Multiple pterygium syndrome: evolution of the phenotype. J Med Genet 24: 733-749.

15. Tsou PM (1977) Embryology of congenital kyphosis. Clin Orthop Relat Res 1 18-25.

16. Chen H, Chang $\mathrm{CH}$, Misra RP, Peters HA, Grijalva NS, et al. (1980) Multiple pterygium syndrome. Am J Med Genet 7: 91-102.
17. Eule JM, Erickson MA, O'Brien MF, Handler M (2002) Chiari I malformation associated with syringomyelia and scoliosis: a twenty-year review of surgical and nonsurgical treatment in a pediatric population. Spine 27: 1451-1455.

18. Wu L Qiu Y, Wang B, Zhu ZZ, Ma WW (2010) The left thoracic curve pattern a strong predictor for neural axis abnormalities in patients with "idiopathic" scoliosis. Spine 35 : 182-185.

19. Davids JR, Chamberlin E, Blackhurst DW (2004) Indications for magnetic resonance imaging in presumed adolescent idiopathic scoliosis. J Bone Joint Surg Am 86: 2187-2195.

20. Blake NS, Lynch AS, Dowling FE (1986) Spinal cord abnormalities in congenital scoliosis. Ann Radiol 29: 377-379.

21. Belmont PJ, Kuklo TR, Taylor KF, Freedman BA, Prahinski JR, et al. (2004) Intraspinal anomalies associated with isolated congenital hemivertebra: the role of routine magnetic resonance imaging. J Bone Joint Surg Am 86: 1704-1710.

22. Robinson LK, Brien NC, Puckett MC, Cox MA (1987) Multiple pterygium syndrome: a case complicated by malignant hyperthermia. Clin Genet 32: 5-9. 